Bacteria were used in two studies of the effects of low doses of radiation. Marcovich (Institut du Radium, France) had examined the induction of lysogenic bacteria, and concluded that the passage of a single ionizing particle through a cell was sufficient to bring about this effect. M. Demerec (Carnegie Institute of Washington, United States) made use of three biochemical mutations which occurred spontaneously with very low frequency, so that the genetic effects of doses as low as $8.5 \mathrm{r}$. could be assessed. In all three cases, the number of mutations induced was proportional to the dose at low doses, though the doses required to produce a given frequency of mutations differed for the three mutations chosen for study.

New observations on the genetic effects of ionizing radiation on mice were reported by W. L. Russell (Oak Ridge, United States). These have confirmed his previous report that if a dose in the range 200 $600 \mathrm{r}$. was delivered at $80 \mathrm{r}$./min. more mutations were induced in spermatogonia than if the same dose was delivered continuously as 'ehronic' irradiation at $90 \mathrm{r}$. /week or less. This did not apply to mutations induced in spermatozoa. The genetic effect of a single dose on oocytes was greater than on spermatogonia, whereas the reverse was true of chronic irradiation. L. B. Russell (Oak Ridge, United States) reported that chronic irradiation was less effective than an acute dose, delivered to embryos in their most sensitive stage, in bringing about still-births and neonatal deaths.

Various tests of radiation damage were used by L. J. Cole (Naval Radiobiological Defense Labora. tory, United States) in comparing effects of single exposures and 'chronic' or fractionated radiation. Single doses were less effective in inducing leukæmia or shortening the life span; but the effects of chronic irradiation doses on the fertility of female mice were much lower than were single doses. The effect of as little as $25 \mathrm{r}$. in a single dose could be detected in weanlings. The injection of bone marrow, which protected against the lethal effects of $800 \mathrm{r}$., did not protect against loss of fertility.

Immunological aspects of tissue transplantation after $300 \mathrm{r}$. of X-rays had been studied by $\mathrm{P}$. C. Koller and S. Doak (Chester Beatty Research Institute, Great Britain). After fifty days, the immune response of some 'chimaeras' had reverted to the host type, others had retained the immunity of the donor, and yet others gave a mixed response.

This very brief account of the subjects discussed at the symposium should make it evident how wide a range of materials, and how many different approaches, are being used in attempts to clarify some of the outstanding problems in radiation biology. The proceedings of the symposium are to be published as a supplement to the International Journal of Radiation Biology.
TikVah AlPer

\title{
NUCLEAR FORCES AND THE FEW-NUCLEON PROBLEM
}

$\mathrm{M}$ ORE than 250 nuclear physicists, including about 100 delegates from fifteen countries overseas, attended the international conference, which was held at the Physics Department, University College, London, during July 8-11. A conference on the behaviour of light nuclei had not taken place for several years and was initiated by physicists at Los Alamos and University College. As was stressed by Prof. H. S. W. Massey (University College), who opened the conference, although the original intention was to emphasize the few-nucleon problem, discussion of nuclear forces had inevitably to be included.

The conference consisted of five main sessions, the first and longest being entirely devoted to the primary two-nucleon interaction. In this session review papers were given by Profs. R. E. Marshak (Rochester), R. Wilson (Harvard), K. A. Brueckner (Pennsylvania) and G. F. Chew (Berkeley) on both the experimental and theoretical status of the problem. Interest centred on many sets of measurements, including triple scattering and correlation experiments, as well as more accurate cross-section measurements at various energies and on their interpretation in terms of the $S$-matrix. Prof. G. Breit (Yale) presented an extensive search for phase-shift fits to the scattering data up to $340 \mathrm{MeV}$., while comparison was made with phase-shifts derived from various phenomenological potentials by Prof. Marshak. There was discussion on both the necessity and theoretical justification for including spin-orbit and other velocity-dependent potentials in the two-body force. The experimental papers were concerned with recent triple scattering measurements in $p-p$ scattering at Rochester and Harwell and with $p-p$ angular distributions at Minnesota. Also $n-p$ angular dis- tributions and polarization measurements from 20-120 MeV. were reported by Dr. J. J. Thresher (Harwell).

Dr. J. Iwadare (Kyoto) summarized the recent work done in Japan on the meson-theoretical twonucleon interaction and its comparison with experimental data. This was followed by Prof. Chew's paper, which reviewed recent work on the meson field-theoretical approach to the two-body problem starting from the Mandelstam conjecture on the analytical form of the scattering amplitude. The inclusion of the pion-pion interaction within the context of dispersion relations seems to be the next step in the long struggle to obtain meaningful results from the meson theory of nuclear forces proposed by Yukawa in 1935 .

Prof. Yukawa was chairman for the beginning of the second session, on the scattering of nucleons by light nuclei at high energy. A review paper on the impulse approximation by Dr. H. McManus (Chalk River) was followed by applications of this approach to the $n-d$ and $p-d$ case by Drs. L. Castillejo (Birmingham), R. Phillips (Harwell) and by Japanese workers. The problem was examined from the point of view of dispersion theory in a paper by Goldberger, Halpern and Blankenbecler (Princeton), and corrections due to multiple scattering were considered by Prof. R. J. Glanber (Harvard). In this session there were reports by Drs. A. M. Cormack, T. C. Griffith and G. Huxtable on experiments done at Harvard, University College and Harwell respectively on $p-d$ and $p-\alpha$ scattering at energies between 50 and $150 \mathrm{MeV}$.

The session on photonuclear reactions with light nuclei opened with a review by Dr. D. Dixon 
(Glasgow). Other papers were presented by Profs. G. Breit, A. Klein (Pennsylvania) and Dr. Iwadare. The effect on the theory of the photodisintegration of the deuteron of the spin-orbit force within the $n-p$ system stimulated much discussion.

The fourth session of the conference was devoted to the question of binding energies and elastic scattering of light nuclei at low energies. The review papers were given by Prof. H. S. W. Massey and Dr. P. G. Burke (University of London) on the threebody problem and on the elastic scattering of nucleons by alpha-particles. There were also two invited papers from Los Alamos: Dr. L. Cranberg reported on experimental results concerning total and differential cross-sections and also polarization measurements in the scattering of low-energy neutrons from ${ }^{2} \mathrm{D},{ }^{3} \mathrm{H}$ and ${ }^{3} \mathrm{He}$, while Dr. L. Rosen reported on charged particle scattering from ${ }^{2} \mathrm{D}$ and ${ }^{3} \mathrm{H}$ at energies up to about $20 \mathrm{MeV}$. These experiments led to several new checks on charge symmetry and time reversal invariance.

The contributed papers included one on the ground-state energy of the triton, by Prof. J. M. Blatt (Sydney). Using the Gammel-Thaler potential, no bound state was found for reasonable trial wave functions. Among other papers from Los Alamos, Dr. J. L. Gammel gave a preliminary account of attempts to integrate the $n-d$ problem numerically and discussed the feasibility of spin-correlation experiments using ${ }^{4} \mathrm{He}$ as an analyser.

Polarization measurements in $n-d$ and $p-d$ scattering at low energies, reported by Dr. H.-J. Gerber (Zurich) and Dr. R. E. Segel (North-western University), indicate that there is very little polarization at about $4 \mathrm{MeV}$. A contribution from Dr. N.
Vlassov (Moscow), read by Dr. I. A. Baz, on the interaction of protons and deuterons with light nuclei ended this session.

The last session, with a title "Reactions Involving Four or More than Four Nucleons", was opened by Dr. B. H. Bransden (Glasgow) with his paper on the collisions of neutrons and of deuterons with ${ }^{3} \mathrm{H}$ and ${ }^{3} \mathrm{He}$. This paper was followed by a number of papers involving the application of the resonating group structure method to binding energy and scattering calculations. This method, as did the impulseapproximation method at higher energies in a previous session, aroused considerable controversy during the discussion. Nevertheless, it was felt that some progress had been made, since one type of mixture of exchange forces does seem to be simulating the exact force in more than one situation involving light nuclei.

Prof. G. Skornyakov (Moscow) then gave his paper on $n-d$ scattering in the zero-range force approxima tion, this being a contribution to the previous session. The three-particle problem is solved accurately in this limit. He also read a paper by Dr. T. Y. Barit on $p$-T scattering and allied reactions.

The final papers were preceded by a review given by Dr. V. J. Emery (Harwell) of the calculations of the binding energies of nuclei using the Brueckner method. Prof. Brueckner himself also presented a paper on the Hartree-Fock method for strongly interacting systems. The conference concluded with papers by Profs. N. Austern and S. Meshkov (Pittsburgh) on preliminary calculations concerning the structure of ${ }^{6} \mathrm{Li}$ and ${ }^{12} \mathrm{C}$.

\section{T. C. Griffith}

E. A. Power

\section{CHAGAS'S DISEASE}

\begin{abstract}
CHAGAS'S disease or South American trypanoA somiasis, occurring chiefly in Brazil and other countries of South America, is caused by Trypanosoma cruzi and is spread mainly by reduviid bugs. The disease was first discovered and described fully in 1909 from the State of Minas Geraes in Brazil. All the significant observations regarding the causative agent, the vector, mode of transmission and symptoms were made then, by that creative genius, Carlos Chagas. In order to commemorate the fiftieth anniversary of this discovery, an international congress on Chagas's disease was held at Rio de Janeiro during July 5-11.

Foreign delegates from European countries, Israel and the United States of America numbered more than seventy. Approximately 300 others from Brazil and the neighbouring countries of South America also attended. At a short historical session at the Ministry of Education, and Culture on July 4 the life and work and significance of the discoveries made by Chagas were described by various speakers.

The inaugural meeting of the congress was held on July 5 in the National Faculty of Medicine from 9 p.m. until midnight, when addresses were delivered by Prof. Alessandri (Chile), Prof. Lemoigne (Pasteur Institute, Paris), Dr. Candau (director general, World Health Organization), a student in the Medical Faculty, Prof. Moraes, director of the Medical School and dean of the University, and replied to by Prof. Carlos Chagas Filho.
\end{abstract}

The real business of the congress began on the following morning at 9 a.m. and lasted until 6 p.m. in a pavilion within the grounds of the Instituto Oswaldo Cruz. Two or three sessions took place concurrently to discuss the disease in its different aspects. In one of the lecture theatres simultaneous translations from English, French, Spanish, Portuguese and German were provided. Chagas's disease in the American continent was discussed from the pathological aspect and the different forms encountered in the various South American countries described, including the clinical findings on the two human cases thus far reported from the southern United States. Other papers on transmitting agents, animal reservoirs, including the opossum and armadillo, and their relation to the epidemiology of the disease as well as the characters of the human strains, were discussed. Public health questions were reviewed in relation to the geographical distribution of the principal transmitters of the disease which infest human dwellings. The anatomy and respiratory system of Triatoma infestans, the chief vector in Brazil, was described. On the following day the subject discussed in one section was the ætiological agent, with emphasis on the physiology, metabolism and nutrition of the parasite. Electron microscope studies of parasitized cells were included. In another section immunological aspects of the disease, including complement fixation reactions, precipitin and skin tests, were dealt with and the isolation of 